\title{
QUENCHING OF FLAMES BY FLUID ADVECTION
}

\author{
PETER CONSTANTIN, ALEXANDER KISELEV AND LEONID RYZHIK
}

\begin{abstract}
We consider a simple scalar reaction-advection-diffusion equation with ignitiontype nonlinearity and discuss the following question: What kinds of velocity profiles are capable of quenching any given flame, provided the velocity's amplitude is adequately large? Even for shear flows, the answer turns out to be surprisingly subtle.

If the velocity profile changes in space so that it is nowhere identically constant, (or if it is identically constant only in a region of small measure) then the flow can quench any initial data. But if the velocity profile is identically constant in a sizable region, then the ensuing flow is incapable of quenching large enough flames, no matter how much larger is the amplitude of this velocity. The constancy region must be wider across than a couple of laminar propagating front-widths.

The proof uses a linear PDE associated to the nonlinear problem and quenching follows when the PDE is hypoelliptic. The techniques used allow the derivation of new, nearly optimal bounds on the speed of traveling wave solutions.
\end{abstract}

\section{INTRODUCTION}

We consider a mixture of reactants interacting in a region that may have a rather complicated spatial structure but is thin across. A mathematical model that describes a chemical reaction in a fluid is a system of two equations for concentration $n$ and temperature $T$ of the form

$$
\begin{aligned}
& T_{t}+u \cdot \nabla T=\kappa \Delta T+\frac{v_{0}^{2}}{\kappa} g(T) n \\
& n_{t}+u \cdot \nabla n=\frac{\kappa}{\mathrm{Le}} \Delta n-\frac{v_{0}^{2}}{\kappa} g(T) n .
\end{aligned}
$$

The equations (1) are coupled to the reactive Euler equations for the advection velocity $u(x, y, t)$. Two assumptions are usually made to simplify the problem: the first is a constant density approximation [8] that allows to decouple the Euler equations from the system (11) and to consider $u(x, y, t)$ as a prescribed quantity that does not depend on $T$ and $n$. The second assumption is that Le $=1$ (equal thermal and material diffusivities). These two assumptions reduce the above system to a single scalar equation for the temperature $T$. We assume in addition that the advecting flow is unidirectional. Then the system (11) becomes

$$
\begin{aligned}
& T_{t}+A u(y) T_{x}=\kappa \Delta T+\frac{v_{0}^{2}}{\kappa} f(T) \\
& T(0, x, y)=T_{0}(x, y)
\end{aligned}
$$

with $f(T)=g(T)(1-T)$. We are interested in strong advection, and accordingly have written the velocity as a product of the amplitude $A$ and the profile $u(y)$. In this paper we consider

email: const@cs.uchicago.edu, kiselev@math.uchicago.edu,ryzhik@math.uchicago.edu. 
nonlinearity of the ignition type

(i) $f(T)$ is Lipschitz continuous on $0 \leq T \leq 1$,

(ii) $f(1)=0, \exists \theta_{0}$ such that $f(T)=0$ for $0 \leq T \leq \theta_{0}, f(T)>0$ for $T>\theta_{0}$,

(iii) $f(T) \leq T$.

The last condition in (3) is just a normalization. We consider the reaction-diffusion equation (2) in a strip $D=\{x \in \mathbb{R}, y \in[0, H]\}$. Equation (2) may be considered as a simple model of flame propagation in a fluid [3], advected by a shear (unidirectional) flow. The physical literature on the subject is vast, and we refer to the recent review 22 for an extensive bibliography. The main physical effect of advection for front-like solutions is the speed-up of the flame propagation due to the large scale distortion of the front. The role of the advection term in (2) for the front-like initial data was also a subject of intensive mathematical scrutiny recently. Existence of unique front-like traveling waves has been established in [3, 6], and their stability has been studied in [18, 19, 21]. A traveling front is a solution of (2) of the form

$$
T(t, x, y)=U\left(x-c_{A} t, y\right)
$$

such that

$$
\lim _{s \rightarrow-\infty} U(s, y)=1, \lim _{s \rightarrow+\infty} U(s, y)=0, U_{s}(s, y)<0 .
$$

The monotonicity property is not required for traveling wave solutions, but it is always present in the situation we consider. The speed-up of the fronts by advection mentioned above may be quantified as the dependence of the traveling front speed $c_{A}$ on the amplitude $A$. Variational formulas for $c_{A}$ were derived in [10, 11]. The latter work also contains results on the asymptotic behavior of $c_{A}$ when $A$ is small for some classes of shear flows, as well as upper bounds on $c_{A}$ linear in $A$. An alternative approach to quantifying advection effects was introduced by the present authors in [7, 15]. It is based on the notion of the bulk burning rate,

$$
V(t)=\int_{D} T_{t} \frac{d x d y}{H}=\frac{v_{0}^{2}}{\kappa} \int_{D} f(T) \frac{d x d y}{H}
$$

which extends the notion of front speed. We derived lower bounds for long time averages of $V(t)$ which behave like $C A$ for large $A$, with constant $C$ depending on the geometry of the flow. These bounds are valid for a class of flows that we call percolating. They are characterized by infinite tubes of streamlines connecting $\pm \infty$ and include shear flows as a particular case. Our bounds imply the estimate $c_{A} \geq C A$ for traveling waves. Audoly, Berestycki and Pomeau gave a formal argument [1] suggesting that in the case when the shear flow varies on the scale much larger than the laminar flame width, one should have $c_{A} \sim A$. One of the by-products of this paper is a rigorous proof of this conjecture.

Our main goal in the present paper is to consider advection effects for a different physically interesting situation, where initial data are compactly supported. In this case, two generic scenarios are possible. If the support of the initial data is large enough, then two fronts form and propagate in opposite directions. Fluid advection speeds up the propagation, accelerating the burning. However, if the support of the initial data is small, then the advection exposes the initial hot region to diffusion which cools it below the ignition temperature, ultimately extinguishing the flame. 
We consider for simplicity periodic boundary conditions

$$
T(t, x, y)=T(t, x, y+H)
$$

in $y$ and decay in $x$ :

$$
T(t, x, y) \rightarrow 0 \text { as }|x| \rightarrow \infty
$$

We take

$$
\int_{0}^{H} u(y) d y=0 .
$$

A constant non-zero mean can be easily taken into account by translation. We consider the case when the width of the domain is larger than the laminar front width length scale: $H \gg l=\frac{\kappa}{v_{0}}$. We will always assume that initial data $T_{0}(x, y)$ is such that $0 \leq T_{0}(x, y) \leq 1$. Then we have $0 \leq T \leq 1$ for all $t>0$ and $(x, y) \in D$. Moreover, we assume that for some $L$ and $\eta>0$ we have

$$
\begin{aligned}
& T_{0}(x, y)>\theta_{0}+\eta \text { for }|x| \leq L / 2, \\
& T_{0}(x, y)=0 \text { for }|x| \geq L .
\end{aligned}
$$

The main purpose of this paper is to study the possibility of quenching of flames by strong fluid advection in a model (2). The phenomena associated with flame quenching are of great interest for physical, astrophysical and engineering applications. The problem of extinction and flame propagation in the mathematical model (2) under conditions (3), (7), (9), was first studied by Kanel [14] in one dimension and with no advection. He showed that, in the absence of fluid motion, there exist two length scales $L_{0}<L_{1}$ such that the flame becomes extinct for $L<L_{0}$, and propagates for $L>L_{1}$. More precisely, he has shown that there exist $L_{0}$ and $L_{1}$ such that

$$
\begin{aligned}
& T(t, x, y) \rightarrow 0 \text { as } t \rightarrow \infty \text { uniformly in } D \text { if } L<L_{0} \\
& T(t, x, y) \rightarrow 1 \text { as } t \rightarrow \infty \text { for all }(x, y) \in D \text { if } L>L_{1} .
\end{aligned}
$$

In the absence of advection, the flame extinction is achieved by diffusion alone, given that the support of initial data is small compared to the scale of the laminar flame width $l=\kappa / v_{0}$. However, in many applications the quenching is the result of strong wind, intense fluid motion and operates on larger scales. There are few results available for such situations in the framework of the reaction-diffusion model. Kanel's result was extended to non-zero advection by shear flows by Roquejoffre [20] who has shown that (10) holds also for $u \neq 0$ with $L_{0}$ and $L_{1}$ depending, in particular, on $A$ and $u(y)$. The second (propagation) part in (10) was also proved in 21] for general periodic flows. However the interesting question about more explicit quantitative dependence of $L_{0}, L_{1}$ on $A$ and $u(y)$ remained open. Is it possible to quench the initial data that previously lead to an expanding solution by increasing $A$, but not changing the profile? How does this possibility depend on geometry of the profile $u(y)$ ? Anyone who has tried to light a match in the wind has some intuition about this phenomenon. Yet, the mathematical answer turns out to be surprisingly subtle. In this paper, we also limit ourselves to shear flows. We are interested in the understanding the behavior of $L_{0}$ and $L_{1}$ for large $A$. The answer depends strongly on the geometry of the flow. In some cases the maximal extinction size grows $L_{0} \sim A$, and in others even the propagation size $L_{1}$ remains finite as $A$ goes to infinity. In the first case, we will say that $u(y)$ is quenching. 
Definition 1. We say that the profile $u(y)$ is quenching if for any $L$ and any initial data $T_{0}(x, y)$ supported inside the interval $[-L, L] \times[0, H]$, there exists $A_{0}$ such that solution of (ब) becomes extinct:

$$
T(t, x, y) \rightarrow 0 \text { as } t \rightarrow \infty \text { uniformly in } D
$$

for all $A \geq A_{0}$. We call the profile u(y) strongly quenching if the critical amplitude of advection $A_{0}$ satisfies $A_{0} \leq C L$ for some constant $C\left(u, \kappa, v_{0}, H\right)$ (which has the dimension of inverse time).

The key feature that distinguishes quenching from non-quenching velocities is the absence or presence of large enough flat parts in the profile $u(y)$.

Definition 2. We say that the profile $u(y) \in C^{\infty}[0, H]$ satisfies the H-condition if

$$
\text { there is no point } y \in[0, H] \text {, where all derivatives of } u(y) \text { vanish. }
$$

The H-condition guarantees that the operator

$$
u(y) \frac{\partial}{\partial x}-\frac{\partial^{2}}{\partial y^{2}}
$$

is hypoelliptic [12]. The study of existence of smooth fundamental solutions for such operators was initiated by Kolmogorov [16]. Kolmogorov's work with $u(y)=y$ served in part as a motivation for the fundamental result on characterization of hypoelliptic operators of Hörmander [12. The hypoellipticity of the operator (12) plays a key role in our considerations. Our first result is that the $\mathrm{H}$-condition implies strong quenching.

Theorem 1. Let $f(T)$ be an ignition type nonlinearity, and let $u \in C^{\infty}[0, H]$ satisfy the $H$-condition. Then $u(y)$ is strongly quenching. That means that there exists a constant $C\left(u, \kappa, v_{0}, H\right)>0$ that may depend on $H, \kappa, v_{0}$ and $u(y)$ but is independent of $A$ such that

$$
T(t, x, y) \rightarrow 0 \text { as } t \rightarrow \infty \text { uniformly in } D \text {. }
$$

This flame extinction occurs whenever the initial temperature $T_{0}(x, y)$ is supported in an interval $[-L, L] \times[0, H]$, with $L<C\left(u, \kappa, v_{0}, H\right) A$.

The next result shows that a plateau on the order of the laminar front width

$$
l=\kappa / v_{0}
$$

in the profile $u(y)$ prohibits quenching. (And therefore the conditions in Theorem 1 are natural.)

Theorem 2. There exist universal constants $C_{0}, C_{1}>0$, such that, if $u(y)=\bar{u}=$ const for $y \in[a-h, a+h]$ for some $a \in[0, H]$ and $h \geq h_{0}=C_{0} l$, then

$$
T(t, x, y) \rightarrow 1 \text { as } t \rightarrow \infty
$$

uniformly on compact sets, for all $A \in \mathbb{R}$. This flame propagation occurs whenever the initial temperature $T_{0}(x, y)$ satisfying (9) is supported in an interval $[-L, L] \times[0, H]$ with $L \geq C_{1} h_{0}$.

An interesting by-product of the proof of Theorem 2 is an estimate for the speed of traveling front solutions of (2) when the shear flow varies slowly on the scale of the laminar flame width $l$. Let us define

$$
\left|u_{+}\right|_{h_{0}}=\max _{0 \leq y_{0} \leq H}\left\{\min _{y \in\left[y_{0}-h_{0}, y_{0}+h_{0}\right]} u_{+}\right\}
$$


with $h_{0}=C l$ given by Theorem 2, and $u_{+}=\max (u(y), 0)$.

Theorem 3. The speed of the traveling front c satisfies the upper and lower bounds

$$
A\left|u_{+}\right|_{h_{0}} \leq c \leq A\left\|u_{+}\right\|_{\infty}+v_{0} .
$$

The upper bound of Theorem 3 is contained in [7] (it is shown there for KPP type reaction, but this immediately implies the corresponding bound for ignition nonlinearity by a simple application of maximum principle). The left hand side is close to $\left\|u_{+}\right\|_{\infty}$ if $u(y)$ is slowly varying on the scale $h_{0}$. This agrees with the formal prediction of Audoly, Berestycki and Pomeau [1], and also (up to the addition of $v_{0}$ ) with the results of Majda and Souganidis [17] in the homogenization regime $\kappa \rightarrow 0$.

Unlike hypoellipticity, the quenching property is stable to small $L^{\infty}$ perturbations: a small enough plateau (on the scale of the laminar front width $l$ ) does not stop quenching.

Theorem 4. For every $\theta_{0}>0$ in (3) there exists a constant $B>0$ such that, if a profile $u(y)$ satisfies the $H$ condition outside an interval $y \in[a-h, a+h]$ with $h \leq h_{1}=B l$, then it is strongly quenching.

Moreover, the strongly quenching profiles are generic in the following sense:

Theorem 5. The set of all strongly quenching shear flows $u(y)$ contains a dense open set in $C[0, H]$ (here $C[0, H]$ is the space of continuous functions on $[0, H]$ ).

In Section 3 we show that all results on quenching, namely, Theorems 1, 4, 5 extend to the case of full system (1) with Le $\neq 1$.

Finally, in the last section we prove that initial data of sufficiently small size (of the same order as in the case $u(y)=0$ ) will be quenched by any shear flow $A u(y)$.

Acknowledgment. We thank N. Nadirashvili for fruitful discussions, and in particular for suggesting that we look for radially symmetric sub-solutions. We also thank G. Papanicolaou for stimulating discussions. This work partially supported by ASCI Flash Center at the University of Chicago. PC was supported by NSF grant DMS-9802611, AK was supported by NSF grant DMS-9801530, and LR was supported by NSF grant DMS-9971742.

\section{QUENCHING BY A SHEAR FLOW}

We prove Theorem 1 in this section.

Proof of Theorem 1. It suffices to show that there exists some time $t_{0}$ such that

$$
T\left(t_{0}, x, y\right) \leq \theta_{0} \text { for all }(x, y) \in D .
$$

Then it follows from the maximum principle that $T(t, x, y) \leq \theta_{0}$ for all $t \geq t_{0}$, and hence $T$ satisfies the linear advection-diffusion equation

$$
T_{t}+A u(y) T_{x}=\kappa \Delta T .
$$

for $t \geq t_{0}$, which implies (13). We actually show that (16) holds at $t_{0}=\kappa / v_{0}^{2}$ for sufficiently large $A$. Recall that $f(T) \leq T$, and hence $T(t, x, y)$ can be bounded from above using the maximum principle as follows:

$$
T(t, x, y) \leq \Phi(t, x, y) e^{v_{0}^{2} t / \kappa} .
$$


Here the function $\Phi(t, x, y)$ satisfies the linear problem

$$
\begin{aligned}
& \Phi_{t}+A u(y) \Phi_{x}=\kappa \Delta \Phi \\
& \Phi(0, x, y)=T_{0}(x, y) \\
& \Phi(t, x, y)=\Phi(t, x, y+H) .
\end{aligned}
$$

Furthermore, we have

$$
\Phi(t, x, y)=\int_{-\infty}^{\infty} d z G(t, x-z) \Psi(t, z, y)
$$

with the function $\Psi(t, x, y)$ satisfying the degenerate parabolic equation

$$
\begin{aligned}
& \Psi_{t}+A u(y) \Psi_{x}=\kappa \Psi_{y y} \\
& \Psi(0, x, y)=T_{0}(x, y) \\
& \Psi(t, x, y)=\Psi(t, x, y+H)
\end{aligned}
$$

and

$$
G(t, x)=\frac{1}{\sqrt{4 \pi \kappa t}} \exp \left(-\frac{x^{2}}{4 \kappa t}\right) .
$$

We note that if $u(y)$ satisfies the H-condition (11) then the diffusion process defined by (19) has a unique smooth transition probability density. Indeed, the Lie algebra generated by the operators $\partial_{y}$ and $\partial_{t}+u(y) \partial_{x}$ consists of vector fields of the form

$$
\frac{\partial}{\partial y}, \frac{\partial}{\partial t}+u(y) \frac{\partial}{\partial x}, u^{\prime}(y) \frac{\partial}{\partial x}, u^{\prime \prime}(y) \frac{\partial}{\partial x}, \ldots, u^{(n)}(y) \frac{\partial}{\partial x}, \ldots
$$

which span $\mathbb{R}^{2}$ if $u(y)$ satisfies (11). Then the theory of Hörmander [12], and the results of Ichihara and Kunita [13] imply that there exists a smooth transition probability density $p_{A}\left(t, x, y, y^{\prime}\right)$ such that

$$
\Psi(t, x, y)=\int_{\mathbf{R}} d x^{\prime} \int_{0}^{H} d y^{\prime} p_{A}\left(t, x-x^{\prime}, y, y^{\prime}\right) T_{0}\left(x^{\prime}, y^{\prime}\right) .
$$

In particular, the function $p_{A}(t)$ is uniformly bounded from above for any $t>0$ [13]. Then we have

$$
T(t, x, y) \leq e^{v_{0}^{2} t / \kappa}\|\Phi(t)\|_{L_{x, y}^{\infty}} \leq e^{v_{0}^{2} t / \kappa}\|\Psi(t)\|_{L_{x, y}^{\infty}} \leq e^{v_{0}^{2} t / \kappa}\left\|p_{A}(t)\right\|_{L_{x, y}^{\infty}}\left\|T_{0}\right\|_{L_{x, y}^{1}} .
$$

It is straightforward to observe that

$$
p_{A}\left(t, x, y, y^{\prime}\right)=\frac{v_{0}}{A} p_{0}\left(t, \frac{A}{v_{0}} x, y, y^{\prime}\right)
$$

with $p_{0}$ being the transition probability density for (19) with $A=v_{0}$. That is, $p_{0}$ satisfies

$$
\begin{aligned}
& \frac{\partial p_{0}}{\partial t}+v_{0} u(y) \frac{\partial p_{0}}{\partial x}=\kappa \frac{\partial^{2} p_{0}}{\partial y^{2}} \\
& p_{0}\left(0, x, y, y^{\prime}\right)=\delta(x) \delta\left(y-y^{\prime}\right) \\
& p_{0}(t, x, y)=p_{0}(t, x, y+H) .
\end{aligned}
$$

Therefore we obtain

$$
T(t, x, y) \leq e^{v_{0}^{2} t / \kappa} \frac{v_{0}}{A}\left\|p_{0}(t)\right\|_{L_{x, y}^{\infty}}\left\|T_{0}\right\|_{L_{x, y}^{1}} \leq 4 e^{v_{0}^{2} t / \kappa} \frac{v_{0}}{A}\left\|p_{0}(t)\right\|_{L_{x, y}^{\infty}} L H
$$


and in particular at time $t_{0}=\frac{\kappa}{v_{0}^{2}}$ we have

$$
T\left(t_{0}, x, y\right) \leq C \frac{v_{0}}{A}\left\|p_{0}\left(t_{0}\right)\right\|_{L_{x, y}^{\infty}} L H \leq \theta_{0}
$$

as long as

$$
\frac{A}{v_{0}} \geq C \frac{\left\|p_{0}\left(t_{0}\right)\right\|_{L_{x, y}^{\infty} L H}}{\theta_{0}} .
$$

Theorem 11 follows from (20) as explained in the beginning of this Section.

We prove now Theorem $₫$ that shows that a sufficiently small plateau in the profile $u(y)$ is not an obstruction to quenching.

Proof of Theorem 因. Let us define the set

$$
D_{r}=\mathbb{R} \times[a-r, a+r] .
$$

As before, it suffices to show that solution of (18) satisfies

$$
\Phi\left(t_{0}, x, y\right) \leq \frac{\theta_{0}}{e}, t_{0}=\frac{\kappa}{v_{0}^{2}}
$$

and that is what we will do. First, we split the initial data for (19) into two parts: one supported on a strip $D_{h_{1}}$, containing the flat part of $u(y)$, and another supported outside it. We will choose $h_{1}=C_{1} l>h$ such that any solution of (19) that is independent of $x$ and with initial data supported inside $D_{h_{1}}$ will be small at time $t_{0}=\kappa / v_{0}^{2}$. The second part is supported away from the strip $D_{h}$, where $u(y)$ is flat. Therefore for a sufficiently small time it behaves like a solution of (19) with advection satisfying the H-condition.

We choose $h_{1}$ as follows. Let $\phi(t, y)$ be a periodic solution of

$$
\phi_{t}=\phi_{y y}, \phi(0, y)=\phi_{0}(y), 0 \leq \phi_{0}(y) \leq 1
$$

given by

$$
\phi(t, y)=\sum_{j \in Z} \phi_{j}(0) e^{2 i j \pi y / H-4 \kappa t \pi^{2} j^{2} / H^{2}}
$$

Then we have

$$
\left|\phi(t, y)-\frac{\|\phi(0, y)\|_{L_{y}^{1}}}{H}\right| \leq 2 \frac{\|\phi(0, y)\|_{L_{y}^{1}}}{H} \sum_{j \geq 1} e^{-4 \kappa t \pi^{2} j^{2} / H^{2}} .
$$

A simple estimate shows that

$$
\sum_{j \geq 1} e^{-\frac{4 \kappa^{2} \pi^{2} j^{2}}{v_{0}^{2} H^{2}}} \leq C \frac{H v_{0}}{\kappa}
$$

and hence

$$
\left|\phi\left(t_{0}, y\right)-\frac{\|\phi(0, y)\|_{L_{y}^{1}}}{H}\right| \leq C \frac{\|\phi(0, y)\|_{L_{y}^{1}}}{l}, t_{0}=\frac{\kappa}{v_{0}^{2}} .
$$

Therefore we have

$$
\phi\left(t_{0}, x, y\right) \leq \frac{\theta_{0}}{10}
$$


as long as

$$
\|\phi(0, y)\|_{L_{y}^{1}} \leq C_{1} l,
$$

where $C_{1}=\theta_{0} H l /(10(l+C H))$. Let us choose $h_{1}$ so that (23) is automatically verified for initial data supported on $D_{2 h_{1}}$ :

$$
h_{1} \leq C_{1} l / 4
$$

with $C_{1}$ as in (23). Let us assume that the width of the interval $[a-h, a+h]$, on which $u(y)$ is constant satisfies

$$
h \leq \frac{h_{1}}{4}
$$

This condition determines the constant $B$ in the statement of Theorem 4 . We may now split the initial data for (19) as follows:

$$
T_{0}(x, y) \leq \chi_{0}(y)+\psi_{0}(x, y) .
$$

Here the smooth function $\chi_{0}(y)$ is supported in the interval $\left[a-2 h_{1}, a+2 h_{1}\right]$ while the function $\psi_{0}(x, y)$ is supported outside the set $\left[a-h_{1}, a+h_{1}\right]$. Both of these functions satisfy in addition $0 \leq \chi_{0}(y), \psi_{0}(x, y) \leq 1$. Then the function $\Phi(t, x, y)$ satisfies the inequality

$$
\Phi(t, x, y) \leq \chi(t, y)+\psi(t, x, y)
$$

with the functions $\chi$ and $\psi$ satisfying (19) with the initial data $\chi_{0}$ and $\psi_{0}$, respectively. It follows from our choice of $h_{1}$ that

$$
\chi\left(t_{0}, y\right) \leq \frac{\theta_{0}}{10}
$$

so it remains only to estimate $\psi\left(t_{0}, x, y\right)$. We will do it separately for $(x, y)$ inside and outside of the strip $D_{h_{1} / 2}$. For the points $(x, y) \in D_{h_{1} / 2}$ we have:

$$
\begin{aligned}
& \psi(t, x, y)=\int_{\mathbf{R}} d x^{\prime} \int_{0}^{H} d y^{\prime} p_{A}\left(t, x-x^{\prime}, y, y^{\prime}\right) \psi_{0}\left(x^{\prime}, y^{\prime}\right) \leq \int_{\mathbf{R}} d x^{\prime} \int_{\left|y^{\prime}-a\right| \geq h_{1}} d y^{\prime} p_{A}\left(t, x-x^{\prime}, y, y^{\prime}\right) \\
& \leq \mathcal{P}\left\{|W(t)| \geq \frac{h_{1}}{2}\right\} \leq \frac{\theta_{0}}{10}
\end{aligned}
$$

for sufficiently small $t$. Here $W(t)$ is the one-dimensional Brownian motion with diffusivity $\kappa$, and $\mathcal{P}$ denotes probability with respect to it. Thus (21) holds inside $D_{h_{1} / 2}$.

In order to estimate the function $\psi(t, x, y)$ outside $\overline{D_{h_{1} / 2}}$ we introduce a profile $\tilde{u}(y)$ that coincides with $u(y)$ outside of the interval $[a-(1+\delta) h, a+(1+\delta) h], \delta \ll 1$, and satisfies the $\mathrm{H}$-condition on the whole interval $[0, H]$. We define the process $(X(t), Y(t))$ by

$$
d X(t)=u(Y(t)) d t, d Y(t)=\sqrt{2 \kappa} d W(t), X(0)=x, Y(0)=y .
$$

Consider the stopping time $\tau$ which is the first time when $Y(t)$ enters the interval $[a-(1+$ $\delta) h, a+(1+\delta) h]$. Then we have

$$
\begin{aligned}
& \psi(t, x, y) \leq P_{x, y}\left\{(X(t), Y(t)) \in \operatorname{supp} \psi_{0}\right\}=P_{x, y}\left\{(X(t), Y(t)) \in \operatorname{supp} \psi_{0} \mid \tau>t\right\} P_{y}(\tau>t) \\
& +P_{x, y}\left\{(X(t), Y(t)) \in \operatorname{supp} \psi_{0} \mid \tau<t\right\} P_{y}(\tau<t) \\
& \leq P_{x, y}\left\{(\tilde{X}(t), \tilde{Y}(t)) \in \operatorname{supp} \psi_{0} \mid \tau>t\right\} P_{y}(\tau>t)+P_{y}(\tau<t) .
\end{aligned}
$$


Here $P_{x, y}$ denotes probability with respect to the process $(X(t), Y(t))$ starting at $(x, y)$, while $P_{y}$ denotes probability with respect to $Y(t)$ starting at $y$ (recall that $Y(t)$ is independent of $x)$. The process $X(t)$ for $t<\tau$ is identical to the process $(\tilde{X}(t), \tilde{Y}(t))$ defined by (24) with $u(Y)$ replaced by $\tilde{u}(Y)$. Therefore we have

$$
\begin{aligned}
& \psi(t, x, y) \leq P_{x, y}\left\{(\tilde{X}(t), \tilde{Y}(t)) \in \operatorname{supp} \psi_{0} \mid \tau>t\right\} P_{y}(\tau>t)+P_{y}(\tau<t) \\
& \leq P_{x, y}\left\{(\tilde{X}(t), \tilde{Y}(t)) \in \operatorname{supp} \psi_{0}\right\}+P_{y}(\tau<t) .
\end{aligned}
$$

Recall that $(x, y) \notin D_{h_{1} / 2}$ and $h \leq h_{1} / 4$. Therefore the point $y$ is a fixed distance away from the interval $[a-(1+\delta) h, a+(1+\delta) h]$. Hence we may choose $t_{1}<t_{0}$ sufficiently small so that

$$
P_{y}\left(\tau<t_{1}\right) \leq \frac{\theta_{0}}{10}
$$

Furthermore, the function $\tilde{\psi}(t, x, y)=P_{x, y}\left\{(\tilde{X}(t), \tilde{Y}(t)) \in \operatorname{supp} \psi_{0}\right\}$ satisfies (19) with the initial data

$$
\tilde{\phi}(0, x, y)= \begin{cases}1, & (x, y) \in \operatorname{supp} \psi_{0} \\ 0, & (x, y) \notin \operatorname{supp} \psi_{0}\end{cases}
$$

However, $\tilde{u}(y)$ is quenching and thus we may choose $A$ so large that

$$
\tilde{\psi}\left(t_{1}, x, y\right) \leq \frac{\theta_{0}}{10}
$$

Therefore we have at $t=t_{1}$ :

$$
\psi\left(t_{1}, x, y\right) \leq \frac{\theta_{0}}{5}
$$

and hence the same upper bound holds at $t=t_{0}>t_{1}$. Therefore (21) holds also outside $D_{h_{1} / 2}$, and Theorem $\$$ follows. The fact that $u(y)$ is strongly quenching follows from this property of $\tilde{u}(y)$.

Theorem 5 is a simple corollary of Theorem 1.

Proof of Theorem 5. The set of all profiles $u(y)$ satisfying H-condition is dense in $C[0, H]$, so by Theorem 1 the set of strongly quenching profiles is dense. To complete the proof, we will show that if $\tilde{u}(y)$ satisfies H-condition, then there exists $\delta(\tilde{u})$ such that if

$$
\|u(y)-\tilde{u}(y)\|_{C[0, H]}<\delta(\tilde{u})
$$

then $u(y)$ is strongly quenching. From the proof of Theorem 1, we know that there exists a constant $C(\tilde{u})$ such that the solution $\tilde{\Psi}(x, y, t)$ of the equation (19) with advection $A \tilde{u}(y)$ satisfies

$$
\tilde{\Psi}\left(x, y, t_{0}\right) \leq \frac{\theta_{0}}{e}
$$

if the initial data $\tilde{\Psi}(x, y, 0)$ is supported on the interval $[-L, L]$ with $L<C(\tilde{u}) A$ (recall that such inequality implies quenching of $T$ with the same initial data). Let $\Psi(x, y, t)$ be a 
solution of (19) with advection $A u(y)$, and initial data supported in $\left[-L^{\prime}, L^{\prime}\right]$. Then by the Feynman-Kac formula (see, e.g. [9])

$$
\begin{array}{r}
\Psi(x, y, t) \leq P_{x, y}\left(x+A \int_{0}^{t} u(y+W(s)) d s \in\left[-L^{\prime}, L^{\prime}\right]\right) \leq \\
P_{x, y}\left(x+A \int_{0}^{t} \tilde{u}(y+W(s)) d s \in\left[-L^{\prime}-A \delta t, L^{\prime}+A \delta t\right]\right) \leq \tilde{\Psi}(x, y, t),
\end{array}
$$

where $\tilde{\Psi}(x, y, t)$ is the solution of (19) with advection $A u(y)$ and initial data equal to the characteristic function of the interval $\left[-L^{\prime}-A \delta t_{0}, L^{\prime}+A \delta t_{0}\right]$ for $t \leq t_{0}$. Now choose $\delta<$ $C(\tilde{u}) / t_{0}$. Then

$$
\Psi\left(x, y, t_{0}\right) \leq \tilde{\Psi}\left(x, y, t_{0}\right) \leq \frac{\theta_{0}}{e}
$$

provided that $L^{\prime} \leq\left(C(\tilde{u})-\delta t_{0}\right) A$, and hence $u(y)$ is strongly quenching.

\section{QUENCHING FOR A SYSTEM}

All results on quenching for equation (2) proved in the previous section extend directly to the case of the system (1). In this respect, the situation is similar to the case $u(y)=0$, where all results on quenching proved by Kanel [14] for a single equation extend to the system case. We make an assumption $g(T) \leq T$, which is just a normalization and corresponds to the condition $f(T) \leq T$ in the case of a single equation. We take compactly supported initial data for the temperature, while for the concentration we assume $0 \leq n(x, y, 0) \leq 1$. Notice that by the maximum principle, $n(x, y, t) \leq 1$ for every $t$. Therefore, $T$ satisfies

$$
T_{t}+u(y) T_{x}-\kappa \Delta T \leq T
$$

and from this point the analysis proceeds in the same way as for scalar equation (starting from (17)). We summarize the results in

Theorem 6. Assume that $g(T)=0$ for $T \leq \theta_{0}$, and $g(T) \leq T$. Suppose that the velocity profile $u(y) \in C_{0}^{\infty}[0, H]$ satisfies $H$-condition, then it is strongly quenching. The same conclusion holds if the profile $u(y)$ has a plateau of the size $h \leq h_{1}=B l$ (where $B$ is a universal constant). Moreover, the set of all quenching shear flows contains a dense open set in $C[0, H]$.

\section{Flame PROPAGATiON}

We prove Theorem 2 in this section. Let $T(t, x, y)$ be solution of (2) with the initial data as in (7). We will use the following result of Xin [21] that holds for more general types of advection (its version for the shear flow was also proved by Roquejoffre in [20]). Consider

$$
\begin{aligned}
& T_{t}+u(x, y) \cdot \nabla T=\kappa \Delta T+\frac{v_{0}^{2}}{\kappa} f(T) \\
& T(0, x, y)=T_{0}(x, y) \in L^{2}(D)
\end{aligned}
$$

with $u(x, y)$ being periodic in both variables, and $0 \leq T_{0}(x, y) \leq 1$. 
QUENCHING OF FLAMES BY FLUID ADVECTION

Proposition 1 (Xin). Assume that the initial data in (25) are such that

$$
\lim _{|x| \rightarrow \infty} T_{0}(x, y)=0 \text { uniformly in } D
$$

and

$$
T_{0}(x, y)>\theta_{0}+\eta \text { for }|x| \leq L .
$$

Then there exists $L_{1}(\eta, u)$ that depends on $\eta$ and $u(x, y)$ such that if $L \geq L_{1}$ then

$$
\lim _{t \rightarrow \infty} T(x-s t, y, t)= \begin{cases}1, & \text { if } c_{l}<s<c_{r} \\ 0, & \text { if } c_{l}>s \text { or } s>c_{r}\end{cases}
$$

Here $c_{l}$ and $c_{r}$ are the speeds of left and right traveling waves, respectively $\left(c_{l}<0, c_{r}>0\right)$.

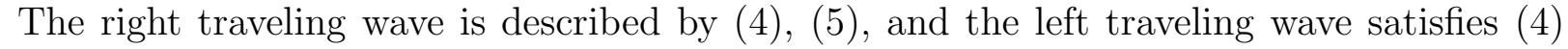
and

$$
\lim _{s \rightarrow-\infty} U(s, y)=0, \lim _{s \rightarrow+\infty} U(s, y)=1, U_{s}(s, y)>0 .
$$

Notice that Theorems 1 and 1 imply an estimate $L_{1} \geq C A$ if $u$ has no flat parts larger than certain critical size. On the other hand, Theorem 2 shows that $L_{1}=h_{0}$ is independent of $A$ if $u$ has a sufficiently large flat part.

Proof of Theorem 2. The proof of Theorem 2 proceeds in several steps. We consider the initial data satisfying (9). First we find $h_{0}$ such that there exists a $C^{2}$ function $\phi(x, y)$ such that $0 \leq \phi<\theta_{0}+\eta$, and

$$
\kappa \Delta \phi+\frac{v_{0}^{2}}{\kappa} f(\phi) \geq 0
$$

and $\phi$ vanishes on the boundary of the disc of radius $h_{0}$ centered at the point $(0, a)$ :

$$
\left.\phi\right|_{\partial \Omega_{0}}=0, \Omega_{0}=B\left((0, a) ; h_{0}\right) .
$$

Then in the system of coordinates that moves with the speed $\bar{u}$ the function $\phi(x, y)$ is a subsolution of (2) in $\Omega_{0}$. Therefore, initial data that start above $\phi$ will not decay to zero. Next we consider the special solution $\Phi(t, x, y)$ of (2) with the initial data given by

$$
\Phi_{0}(x, y)= \begin{cases}\phi(x, y) & (x, y) \in \Omega_{0} \\ 0 & (x, y) \notin \Omega_{0}\end{cases}
$$

We show that $\Phi(t, x, y)$ satisfies (15) and that implies that (15) holds for arbitrary initial data $T_{0} \geq \Phi_{0}$, in particular, such as described in Theorem 2 .

Step 1. Construction of a stationary sub-solution. Choose $\theta_{1}, \theta_{2}$ so that $\theta_{0}+\eta>$ $\theta_{2}>\theta_{1}>\theta_{0}$ and define $f_{1}(T)$ by

$$
f_{1}(T)= \begin{cases}0, & T \leq \theta_{1} \\ \frac{f\left(\theta_{2}\right)\left(T-\theta_{1}\right)}{\theta_{2}-\theta_{1}}, & \theta_{1} \leq T \leq \theta_{2} \\ f(T), & \theta_{2} \leq T \leq 1\end{cases}
$$

The function $f(T)$ is Lipschitz continuous, and hence we may choose $\theta_{1}$ and $\theta_{2}$ so that $f_{1}(T) \leq f(T)$. Therefore if $\phi$ satisfies

$$
\kappa \Delta \phi+\frac{v_{0}^{2}}{\kappa} f_{1}(\phi)=0
$$


then $\phi$ satisfies (26). We are going to construct an explicit radial solution $\phi(r)$ of (28) with the "initial" conditions

$$
\phi(0)=\theta_{2}, \frac{\partial \phi}{\partial r}(0)=0 .
$$

Indeed, $\phi(r)$ is given explicitly by

$$
\phi(r)=\theta_{1}+\left(\theta_{2}-\theta_{1}\right) J_{0}\left(\frac{r v_{0} \sqrt{\alpha}}{\kappa}\right), \alpha=\frac{f\left(\theta_{2}\right)}{\theta_{2}-\theta_{1}} \text { for } r \leq R_{1}=\frac{\kappa \xi_{1}}{v_{0} \sqrt{\alpha}} .
$$

Here $J_{0}(\xi)$ is the Bessel function of order zero, and $\xi_{1}$ is its first zero. Furthermore, we have

$$
\phi(r)=B \ln \frac{r}{R}, \text { for } R_{1} \leq r
$$

with $B$ and $R$ determined by matching (29) and (30) at $r=R_{1}$. Then we get

$$
R=l \xi_{1} \sqrt{\frac{\theta_{2}-\theta_{1}}{f\left(\theta_{2}\right)}} \exp \left[\frac{\theta_{1}}{\left(\theta_{2}-\theta_{1}\right) \xi_{1}\left|J_{0}^{\prime}\left(\xi_{1}\right)\right|}\right]=C l, l=\frac{\kappa}{v_{0}} .
$$

Then $\phi(r)$ satisfies

$$
\begin{aligned}
& \kappa \Delta \phi+\frac{v_{0}^{2}}{\kappa} f(\phi) \geq 0, \phi(r)>0,0 \leq r<R \\
& \phi(R)=0 .
\end{aligned}
$$

Thus we will take the critical size of plateau in the velocity profile to be $2 R$ so that the disc of radius $R$ will fit in.

Step 2. A sub-solution. Let us now assume that $h \geq h_{0}=R$. We make a coordinate change

$$
\xi=x-\bar{u} t
$$

In new coordinates we have a function $T(t, \xi, y)$ that solves

$$
T_{t}+A(-\bar{u}+u(y)) T_{\xi}=\kappa \Delta_{\xi, y} T+\frac{v_{0}^{2}}{\kappa} f(T)
$$

with the initial data given by $(27)$ :

$$
T(0, \xi, y)=\left\{\begin{array}{ll}
\phi(\xi, y), & (\xi, y) \in \Omega_{0}=B\left((0, a) ; h_{0}\right) \\
0, & (\xi, y) \notin \Omega_{0}
\end{array} .\right.
$$

Observe that $\phi(\xi, y)$ satisfies (26) inside $\Omega_{0}$ since $u(y)=\bar{u}$ in $\Omega_{0}$. Moreover, $T(t, \xi, y) \geq \phi(\xi, y)$ on $\partial \Omega_{0}$, where $\phi$ vanishes. Therefore the maximum principle implies that inside $\Omega_{0}$ we have

$$
T(t, \xi, y) \geq \phi(\xi, y) \text { for all } t>0 \text { and }(\xi, y) \in \Omega_{0} .
$$

Note that $T_{h}(t, \xi, y)=T(t+h, \xi, y)$ solves (31) with the initial data

$$
T_{h}(0, \xi, y)=T(h, \xi, y) \geq T(0, \xi, y) .
$$

The inequality in (33) follows from (32) inside $\Omega_{0}$ and the fact that $T(t, x, y) \geq 0$ outside $\Omega_{0}$. Therefore we have

$$
T(t+h, \xi, y)-T(t, \xi, y) \geq 0 \text { for all } h>0, t>0 \text { and }(\xi, y) \in D
$$

and thus the limit

$$
\bar{T}(\xi, y)=\lim _{t \rightarrow \infty} T(t, \xi, y)
$$


exists since $T \leq 1$. Moreover, the standard parabolic regularity implies that $T(t, \xi, y)$ converges to $\bar{T}(\xi, y)$ uniformly on compact sets together with its derivatives up to the second order. Therefore $\bar{T}$ satisfies the stationary problem

$$
(-\bar{u}+u(y)) \bar{T}_{\xi}=\kappa \Delta_{\xi, y} \bar{T}+\frac{v_{0}^{2}}{\kappa} f(\bar{T})
$$

We also have

$$
\bar{T}(\xi, y)>\phi(\xi, y) \text { for }(\xi, y) \in \Omega_{0} .
$$

It is easy to show using the sliding method of Berestycki and Nirenberg [5] that for any $(\xi, y) \in D_{h}$

$$
\bar{T}(\xi, y)>\phi(\xi-r, y)
$$

where the right side is any translation of $\phi$ along the $\xi$ axis. Indeed, assume that there exists the smallest (say, positive) $r$ such that $\bar{T}\left(\xi_{0}, y_{0}\right)=\phi\left(\xi_{0}-r, y_{0}\right)$ at some point $\left(\xi_{0}, y_{0}\right) \in D_{h}$. Then the strong maximum principle implies that $\bar{T}(\xi, y)=\phi(\xi-r, y)$ for all $(\xi, y)$ inside the translate $\Omega_{r}=\Omega_{0}-r e_{1}$ of the disc $\Omega_{0}$. But that contradicts the fact that $\bar{T}(\xi, y)>$ $\phi(\xi-r, y)=0$ on the boundary $\partial \Omega_{r}$. Then (35) implies that

$$
\bar{T}(\xi, a)>\theta_{2}=\sup _{(\xi, y) \in \Omega_{0}} \phi(\xi, y) \text {, for all } \xi \in \mathbb{R} .
$$

The next two lemmas show that (34) and (36) imply that $\bar{T}(\xi, y) \equiv 1$.

Lemma 1. Let $\bar{T}$ be a solution of (31) such that (30) holds. Then we have

$$
\int_{D} d \xi d y f(\bar{T}(x, y))<\infty, \int_{D} d \xi d y|\nabla \bar{T}|^{2}<\infty .
$$

Proof. In order to show that integral of $f(\bar{T})$ is finite we integrate (34) over the set $(-L+$ $\zeta, L+\zeta) \times[0, H]$ with $L$ large and $\zeta \in[0, l]$. We get

$$
\begin{aligned}
& A \int_{0}^{H} d y[-\bar{u}+u(y)][\bar{T}(L+\zeta, y)-\bar{T}(-L+\zeta, y)]=\kappa \int_{0}^{H} d y\left[\bar{T}_{\xi}(L+\zeta, y)-\bar{T}_{\xi}(-L+\zeta, y)\right] \\
& +\frac{v_{0}^{2}}{\kappa} \int_{0}^{H} d y \int_{-L+\zeta}^{L+\zeta} d \xi f(\bar{T}(\xi, y)
\end{aligned}
$$

and average this equation in $\zeta \in[0, l]$ :

$$
\begin{aligned}
& \frac{A}{l} \int_{0}^{l} d \zeta \int_{0}^{H} d y[-\bar{u}+u(y)][\bar{T}(L+\zeta, y)-\bar{T}(-L+\zeta, y)] \\
& =\frac{\kappa}{l} \int_{0}^{H} d y[\bar{T}(L+l, y)-\bar{T}(L, y)-\bar{T}(-L+l, y)+\bar{T}(-L, y)] \\
& +\frac{v_{0}^{2}}{l \kappa} \int_{0}^{l} d \zeta \int_{0}^{H} d y \int_{-L+\zeta}^{L+\zeta} d \xi f(\bar{T}(\xi, y) .
\end{aligned}
$$


Therefore we obtain

$$
\frac{v_{0}^{2}}{\kappa} \int_{0}^{H} d y \int_{-L+l}^{L} d \xi f(\bar{T}(\xi, y)) \leq \frac{4 \kappa H}{l}+2 H A\left[\bar{u}+\|u\|_{\infty}\right]
$$

for all $L$, and hence the first inequality in (37) holds. In order to obtain the second inequality we multiply (34) by $T$ and perform the same integration and averaging as above. This leads to

$$
\begin{aligned}
& \frac{A}{2 l} \int_{0}^{l} d \zeta \int_{0}^{H} d y[-\bar{u}+u(y)]\left[\bar{T}^{2}(L+\zeta, y)-\bar{T}^{2}(-L+\zeta, y)\right] \\
& =\frac{\kappa}{2 l} \int_{0}^{H} d y\left[\bar{T}^{2}(L+l, y)-\bar{T}^{2}(L, y)-\bar{T}^{2}(-L+l, y)+\bar{T}^{2}(-L, y)\right] \\
& +\frac{1}{l} \int_{0}^{l} d \zeta \int_{0}^{H} d y \int_{-L+\zeta}^{L+\zeta} d \xi\left[\frac{v_{0}^{2}}{\kappa} T f(\bar{T})+\kappa|\nabla T|^{2}\right]
\end{aligned}
$$

and then the second inequality in (37) follows from (38) and the first inequality in (37).

Lemma 2. The limit function $\bar{T}(\xi, y) \equiv 1$

Proof. Notice that $\bar{T}$ can not achieve local minima in $D$ as follows from the maximum principle. Therefore if we define $\mu_{\alpha, \beta}=\min _{\alpha \leq \xi \leq \beta, 0 \leq y \leq H} \bar{T}(\xi, y)$ and $\mu(\alpha)=\min _{0 \leq y \leq H} \bar{T}(\alpha, y)$ then $\mu_{\alpha, \beta}=\mu(\alpha)$ or $\mu_{\alpha, \beta}=\mu(\beta)$. Furthermore, if $\bar{T}(\xi, y)=1$ at some point, then $\bar{T}(\xi, y) \equiv 1$ everywhere by the strong maximum principle. In particular, if $\mu(0)=1$, then $\bar{T}(\xi, y) \equiv 1$. Therefore we consider only the case that $\mu(0)<1$ and argue by contradiction. We have either $\mu(\xi)<\mu(0)$ for any $\xi>0$, or for any $\xi<0$. Otherwise the minimum of $\bar{T}$ over the set $[-\xi, \xi] \times[0, H]$ would be achieved inside. Let us assume without loss of generality that $\mu(\xi)<\mu(0)$ for any $\xi>0$. Consider

$$
\delta=\min \left(\frac{1-\mu(0)}{2}, \frac{\theta_{2}-\theta_{0}}{2}\right) .
$$

For any $\xi>l$, we have three options.

1. $\bar{T}(\xi, y) \in[\mu(\xi), \mu(\xi)+\delta]$ for any $y \in[0, H]$, and $\mu(\xi) \geq \theta_{0}+\delta$. In this case, by definition of $\delta$, properties (3) of $f$, and since $\mu(\xi) \leq \mu(0)<1$, we have

$$
\int_{0}^{H} f(\bar{T}(\xi, y)) d y \geq C H
$$

2. $\mu(\xi)<\theta_{0}+\delta$. Inequality (36) implies that there exist $y_{1}, y_{2}$ such that $\bar{T}\left(\xi, y_{1}\right)=\theta_{2}-\delta$, $\bar{T}\left(\xi, y_{2}\right)=\theta_{2}$. Then

$$
\left|\int_{y_{1}}^{y_{2}} f(\bar{T}(\xi, y)) d y\right| \geq C\left|y_{2}-y_{1}\right|
$$


QUENCHING OF FLAMES BY FLUID ADVECTION

$$
\int_{y_{1}}^{y_{2}}\left|\bar{T}_{y}\right|^{2} d y \geq\left|y_{2}-y_{1}\right|^{-1}\left(\int_{y_{1}}^{y_{2}}\left|\bar{T}_{y}\right| d y\right)^{2} \geq \delta^{2}\left|y_{2}-y_{1}\right|^{-1} .
$$

Therefore,

$$
\int_{H}^{0} f(\bar{T}(\xi, y)) d y \int_{y_{1}}^{y_{2}}\left|\bar{T}_{y}\right|^{2} d y \geq C \delta^{2}
$$

in this case.

3. The remaining option is that $\mu(\xi) \geq \theta_{0}+\delta$, and there exists $y \in[0, H]$ such that $\bar{T}(\xi, y)>$ $\mu(\xi)+\delta$. In this case, an argument identical to the reasoning of option two leads to the same bound (39).

Overall, we see that for any $\xi<l$,

$$
\int_{0}^{H}\left(f\left(\bar{T}(\xi, y)+|\nabla \bar{T}|^{2}(\xi, y)\right) d y \geq C,\right.
$$

where $C$ depends only on $H$ and $\delta$. But this contradicts directly Lemma 1 .

Using Proposition 1, we can now complete the proof of Theorem 2. Notice that even though we showed $\bar{T}(\xi, y) \equiv 1$, we still have to show the limiting function is the same in the original coordinates. Lemma 2 implies that there exists a time $t_{0}$ so that for all $\xi \in$ $\left[0, L_{0}\left(\theta_{2}-\theta_{0}, A u(y)\right)\right]$ and all $y \in[0, H]$ we have

$$
T\left(t_{0}, \xi, y\right) \geq \theta_{0}+\eta \text {. }
$$

Here $L_{0}(\eta, v)$ is defined by Proposition 1. Then we may apply Proposition 11 with $s=0$ in the original coordinates $(x, y)$, and initial data $T\left(t_{0}, x, y\right)$, and get (15). The fact that $c_{l}<0<c_{r}$ follows from (8) and, for instance, results of [3].

The sub-solution we constructed in Theorem 2 is also useful for a proof of Theorem 3 . As mentioned in the Introduction, we only need to show the lower bound, the upper bound is contained in [7]. We begin the proof with two auxiliary lemmas.

Lemma 3. Let $u(y)$ be a function that is constant on an interval:

$$
u(y)=\bar{u}, y \in[a-h, a+h]
$$

that may or may not satisfy the mean zero condition (8). Assume that $h \geq h_{0}$ with $h_{0}$ given in Theorem 圂. Then we have $c_{l}(u) \leq \bar{u} \leq c_{r}(u)$ (recall that $c_{l}(u)$ and $c_{r}(u)$ are the velocities of unique left and right traveling fronts).

Proof. Let $T_{0}$ be an initial data as in Theorem 2 such that

$$
T_{0}(x, y) \geq \begin{cases}\phi(x, y), & (x, y) \in \Omega_{0}=B\left((0, a) ; h_{0}\right) \\ 0, & (\xi, y) \notin \Omega_{0}\end{cases}
$$

with the function $\phi(x, y)$ constructed in the proof of Theorem 2 and given explicitly by (29) and (30). Then we have

$$
T(t, x-\bar{u} t, y) \geq \phi(x, y)
$$

and therefore Proposition 1 implies that $c_{l} \leq \bar{u} \leq c_{r}$ because $T(t, x-\bar{u} t, y)$ may not go to zero as $t \rightarrow \infty$. 
Let $y_{0}$ be a point at which $\left|u_{+}\right|_{h_{0}}$ is achieved: $u(y) \geq\left|u_{+}\right|_{h_{0}}$ for $y \in\left[y_{0}-h_{0}, y_{0}+h_{0}\right]$. We define now a new velocity field

$$
v(y)= \begin{cases}u(y), & y \notin\left[y_{0}-h_{0}, y_{0}+h_{0}\right] \\ \left|u_{+}\right| h_{0}, & y \in\left[y_{0}-h_{0}, y_{0}+h_{0}\right]\end{cases}
$$

so that $u(y) \geq v(y)$.

Lemma 4. Let $u(y) \geq v(y)$ be two velocity fields. Then the corresponding right traveling front speeds satisfy $c_{r}(u) \geq c_{r}(v)$. Similarly, if $u(y) \leq v(y)$, then $c_{l}(u) \leq c_{l}(v)$.

Proof. Let $T^{u, v}(t, x, y)$ be solutions of

$$
T_{t}^{u}+A u(y) T_{x}^{u}=\kappa \Delta T^{u}+\frac{v_{0}^{2}}{\kappa} f\left(T^{u}\right)
$$

and

$$
T_{t}^{v}+A v(y) T_{x}^{v}=\kappa \Delta T^{v}+\frac{v_{0}^{2}}{\kappa} f\left(T^{v}\right)
$$

with the same initial data

$$
T^{u}(0, x, y)=T^{v}(0, x, y)=T_{0}(x, y) .
$$

The function $T_{0}(x, y)$ is assumed to be front-like and monotonic. That is, $T_{0}(x, y)=1$ for $x \leq-L, T_{0}(x, y)=0$ for $x \geq L$ and

$$
\frac{\partial T_{0}}{\partial x}(x, y) \leq 0
$$

Then by maximum principle applied to the equation for $\partial T^{u, v} / \partial x$ we have

$$
\frac{\partial T^{u, v}}{\partial x}(t, x, y) \leq 0 \text { for all } t>0
$$

and hence

$$
T_{t}^{v}+A u(y) T_{x}^{v}-\kappa \Delta T^{v}-\frac{v_{0}^{2}}{\kappa} f\left(T^{v}\right)=A(u(y)-v(y)) T_{x}^{v} \leq 0 .
$$

Therefore by maximum principle we have

$$
T^{u}(t, x, y) \geq T^{v}(t, x, y) .
$$

However, by results of [21, 20] similar to Proposition 11 but for the front-like initial data we have

$$
\lim _{t \rightarrow \infty} T^{v}(x-s t, y, t)= \begin{cases}1, & \text { if } s<c_{r}(v) \\ 0, & \text { if } s>c_{r}(v)\end{cases}
$$

and similarly for $T^{u}$. Then $c_{r}(v)>c_{r}(u)$ would be incompatible with (41), and hence $c_{r}(v) \leq$ $c_{r}(u)$. Naturally, an analogous result is true for left traveling fronts.

Proof of Theorem 3. In order to finish the proof of Theorem 3 we apply Lemma 4 to $v(y)$ given by (40), and observe that $c_{r}(v)$ satisfies the estimate in Theorem 3 according to Lemma 3.

Finally, we remark that in the situation of Theorem 2 strong advection not only does not quench the flame, but has directly opposite effect, according to Theorem 3 and Proposition 1 . 
Corollary 1. Let $u(y)=\bar{u}=$ const for $y \in[a-h, a+h]$ for some $a \in[0, H]$, and $h$ is larger than or equal to the critical size $h_{0}$. Then, provided that the size of initial data $L \geq h_{0}$, we have

$$
\lim _{t \rightarrow \infty} T(x-s t, y, t)= \begin{cases}1, & \text { if } c_{l}<s<c_{r} \\ 0, & \text { if } c_{l}>s \text { or } s>c_{r}\end{cases}
$$

Moreover, $c_{r} \geq\left|u_{+}\right|_{h_{0}}$ and $c_{l} \leq-\left|u_{-}\right|_{h_{0}}$.

Proof. The first statement is a direct corollary of Proposition 1 and Theorem 2 . The second statement is the content of Theorem 0 .

\section{A LOWER BOUND FOR THE QUENCHING SIZE}

Recall that the burning rate is defined by

$$
V(t)=\int_{D} T_{t}(x, y) \frac{d x d y}{H} .
$$

We also say that nonlinearity $f(T)$ is of concave KPP class if

$$
f(0)=f(1)=0, f(T)>0 \text { for } 0<T<1, f^{\prime \prime}(T)<0 .
$$

We have previously shown [7] that for such nonlinearities and for front-like initial conditions the burning rate in the presence of any advection is bounded from below by $C v_{0}$, more precisely:

$$
V(t) \geq C v_{0}\left(1-e^{-C v_{0}^{2} t / \kappa}\right) .
$$

The physical meaning of (42) is that no advection may slow up the burning significantly. We now show that similarly there is a fixed size of initial data that is quenched by any shear flow. That means that no shear flow may help prevent quenching of initial data with a fixed small support. Let $T(t, x, y)$ be solution of

$$
T_{t}+u(y) T_{x}=\kappa \Delta T+\frac{v_{0}^{2}}{\kappa} f(T)
$$

with the initial data $T(0, x, y)=T_{0}(x, y)$ and periodic boundary conditions (6).

Proposition 2. There exists a constant $C>0$ that depends only on the non-linearity $f(T)$ such that given any initial data $T_{0}$ with

$$
\int_{D} T_{0}(x, y) d x d y \leq C l^{2}, l=\frac{\kappa}{v_{0}}
$$

we have

$$
T(t, x, y) \rightarrow 0 \text { uniformly in } D \text { as } t \rightarrow+\infty
$$

for all $u(y) \in C[0, H]$.

Proof. We will show that solution of (43) with $f(T)$ replaced by a KPP nonlinearity $\tilde{f}(T)=$ $M T(1-T)$ and the same initial data drops below $\theta_{0}$ before time $t_{0}=\kappa / v_{0}^{2}$. That will imply (44). Choose $M$ such that $f(T) \leq \tilde{f}(T)=M T(1-T)$ (such $M$ exists since $f$ is Lipschitz continuous) and let $\tilde{T}$ be the solution of

$$
\tilde{T}_{t}+u(y) \tilde{T}_{x}=\kappa \Delta \tilde{T}+\frac{v_{0}^{2}}{\kappa} \tilde{f}(\tilde{T})
$$


with initial data $T_{0}$ and periodic boundary conditions (6). Let us also define

$$
\tilde{V}(t)=\int_{D} \tilde{T}_{t}(x, y) \frac{d x d y}{H}=\frac{M v_{0}^{2}}{\kappa} \int_{D} \tilde{T}(1-\tilde{T}) \frac{d x d y}{H} .
$$

Note that $\tilde{T}(t, x, y) \geq T(t, x, y)$ and hence Proposition 2 follows from the following Lemma.

Lemma 5. There exists a constant $C>0$ that depends only on the non-linearity $f(T)$ such that given any initial data $T_{0}$ with

$$
\int_{D} T_{0}(x, y) d x d y \leq C l^{2}, l=\frac{\kappa}{v_{0}},
$$

and any $u(x, y) \in C^{1}(D)$, there exists a time $t_{1}<t_{0}$ such that $\tilde{T}(t, x, y) \leq \theta_{0}$ for all $(x, y) \in D$.

Proof. The following inequality holds for $\tilde{V}(t)$ :

$$
\tilde{V}(t)+\frac{\kappa}{M v_{0}^{2}} \frac{d \tilde{V}(t)}{d t} \geq 2 \kappa \int_{D}|\nabla \tilde{T}|^{2} \frac{d x d y}{H} .
$$

We define the set

$$
\mathcal{S}(t)=\left\{y \in[0, H]: \exists x \in \mathbb{R} \text { such that } T(t, x, y) \geq \theta_{0} / e^{2 M}\right\} \subset[0, H] .
$$

Observe that there exists a constant $\delta>0$ that depends only on $M$ and $\theta_{0}$ such that if the Lebesgue measure $|\mathcal{S}(\tau)| \leq \delta l$ at some time $\tau \in\left[0, t_{0} / 2\right]$, then $\left\|\tilde{T}\left(t_{0}\right)\right\|_{L^{\infty}} \leq \theta_{0}$. Indeed, we have then

$$
\tilde{T}(\tau, x, y) \leq \chi(y)+\psi(x, y),\|\chi(y)\| \leq \delta l, \psi(x, y) \leq \theta_{0} / e^{2 M} .
$$

Therefore we have with $C$ as in (22)

$$
T\left(t_{0}, x, y\right) \leq e^{M}\left[(C+1) \delta+\frac{\theta_{0}}{e^{2 M}}\right] \leq \theta_{0}
$$

for a sufficiently small $\delta$. Hence it suffices to consider the case when $|\mathcal{S}(t)| \geq \delta l$ for all $t \in\left[0, t_{0} / 2\right]$. We claim that then at any time $t \in\left[0, t_{0} / 2\right]$ we have

$$
\int_{D}|\nabla \tilde{T}|^{2} d x d y \int_{D} \tilde{f}(\tilde{T}) d x d y \geq C l^{2}
$$

with the constant $C$ depending only on $M$ and $\theta_{0}$. This may be proved similarly to Lemma 2 in [7]. Therefore we obtain

$$
\tilde{V}(t)+\frac{\kappa}{M v_{0}^{2}} \frac{d \tilde{V}(t)}{d t} \geq \frac{2 C v_{0}^{2} l^{2}}{H^{2} \tilde{V}(t)} .
$$

Thus we have

$$
\tilde{V}(t) \geq \frac{C v_{0} l}{H} \text { for } t_{0} / 2 \leq t \leq t_{0}
$$

Therefore

$$
\int_{D} \tilde{T}\left(t_{0}, x, y\right) \geq \int_{D} T_{0}(x, y) d x d y+C \kappa t_{0} .
$$


However, we have an a priori bound

$$
\int_{D} \tilde{T}\left(t_{0}, x, y\right) d x d y \leq e^{M} \int_{D} T_{0}(x, y) d x d y
$$

which contradicts the previous inequality if

$$
\int_{D} T_{0}(x, y) d x d y \leq \frac{C \kappa t_{0}}{e^{M}-1}=C_{M} l^{2} .
$$

Therefore $T(t, x, y)$ has to drop below $\theta_{0}$ if the initial data satisfies (46) and Lemma 5 is proved.

Proposition 2 then follows from Lemma 5.

Remark. The uniform quenching size in Proposition 2 is optimal. Indeed, the subsolution we have constructed in Section 4 for shear flows with a flat part has $L^{1}$ norm $C l^{2}$, and thus one cannot expect that initial data with $L^{1}$-norm larger than $C l^{2}$ are quenched by all shear flows.

\section{REFERENCES}

[1] Audoly, B., Berestycki, H. and Pomeau, Y., Rèaction diffusion en ècoulement stationnaire rapide, to appear in C.R. Acad. Sci., 2000.

[2] H. Berestycki and F. Hamel, Front propagation in periodic media, Preprint, 2000.

[3] H. Berestycki, B. Larrouturou and P. L. Lions, Multi-dimensional traveling wave solutions of a flame propagation model, Arch. Rational Mech. Anal., 111, 1990, 33-49.

[4] H. Berestycki, B. Larrouturou and J.-M. Roquejoffre, Stability of traveling fronts in a model for flame propagation I: linear stability, Arch. Rational Mech. Anal., 117, 1992, 97-117.

[5] Berestycki, H. and Nirenberg, L., Some qualitative properties of solutions of semilinear elliptic equations in cylindrical domains, in Analysis etc. (volume dedicated to J. Moser), P. Rabinowitz, E. Zehnder, Eds., Academic Press, NY, 1990, 115-164.

[6] H. Berestycki and L. Nirenberg, Traveling fronts in cylinders, Annales de l'IHP, Analyse non linéare, 9, 1992, 497-572.

[7] P. Constantin, A. Kiselev, A. Oberman and L. Ryzhik, Bulk burning rate in passive-reactive diffusion, to appear in Arch. Rat. Mech. Anal., 2000.

[8] P.Clavin and F.A.Williams, Theory of pre-mixed flame propagation in large-scale turbulence, Jour. Fluid. Mech., 90, 1979, 589-604.

[9] M. Freidlin, Functional integration and partial differential equations, Annals of Mathematics Studies, 109, Princeton University Press, Princeton, 1985.

[10] F. Hamel, Formules min-max pour les vitesses d'ondes progressives multidimensionnelles, Ann. Fac. Sci. Toulouse, 8, 1999, 259-280.

[11] S. Heinze, G. Papanicolaou and A. Stevens, Variational principles for propagation speeds in inhomogeneous media, Preprint, 1999.

[12] Hörmander, L., Hypoelliptic second order differential equations, Acta math., 119, 1967, 147-171.

[13] Ichihara, K. snd Kunita, H., A classification of the second order degenerate elliptic operators and its probabilistic characterization, Z. Wahrscheinlichkeitstheorie verw., 30, 1974, 235-254.

[14] Kanel, Ya., Stabilization of solutions of the Cauchy problem for equations encountered in combustion theory, Mat. Sbornik, 59, 1962, 245-288.

[15] Kiselev, A. and Ryzhik, L., Enhancement of the Traveling Front Speeds in Reaction-Diffusion Equations with Advection, Preprint, 2000.

[16] Kolmogorov, A.N., Zufällige Bewegungen, Ann. of Math. (2), 35 (1934), 116-117.

[17] A. Majda and P. Souganidis, Large scale front dynamics for turbulent reaction-diffusion equations with separated velocity scales, Nonlinearity, 7, 1994, 1-30. 
[18] J.-F. Mallordy and J.-M. Roquejoffre, A parabolic equation of the KPP type in higher dimensions, SIAM J. Math. Anal., 26, 1995, 1-20.

[19] Roquejoffre, J.-M., Stability of traveling fronts in a model for flame propagation II, Arch. Rat. Mech. Anal. 117, 1992, 119-153.

[20] Roquejoffre, J.-M.,Eventual monotonicity and convergence to traveling fronts for the solutions of parabolic equations in cylinders, Annal. Inst. Poincare, Analyse Nonlinéare, 14, 1997, 499-552.

[21] J. Xin, Existence and nonexistence of traveling waves and reaction-diffusion front propagation in periodic media, Jour. Stat. Phys., 73, 1993, 893-926.

[22] J. Xin, Analysis and modeling of front propagation in heterogeneous media, SIAM Review, SIAM Review, 42, 2000, 161-230.

Department of Mathematics, University of Chicago, Chicago IL 60637 